\title{
Financial Literacy Levels amongst Salaried Women: Do they have a Bearing on their Financial Behaviour?
}

\author{
Ravneet Kaur ${ }^{1 *}$ and Rajiv Kumar Maheshwary ${ }^{2}$ \\ 1Ph.D. Research Scholar, School of Management and Commerce, RIMT University, Mandi Gobindgarh, Punjab - \\ 147301, India; ravneetkaurmgt@gmail.com \\ 2Professor cum Dean, School of Management and Commerce, RIMT University, Mandi Gobindgarh, Punjab - \\ 147301, India, rajivkm.Idh@gmail.com
}

\begin{abstract}
The introduction of a wide array of financial instruments, alongside increased complexity has rendered it crucial for financially vulnerable groups like women to understand the real premises on which they are centered as opposed to being simply aware of them. The study using statistical tools including Descriptives, ANOVA, Chi-Square, Factor analysis conducted during October 2020 - January 2021 assessed financial literacy levels among salaried women in Ludhiana City and its effect on financial behaviour, found the average financial literacy score to be $68 \%$, being higher than OECD financial literacy survey score of $60.48 \%$. Additionally, financial literacy had significant effect on respondents' subsequent financial behaviour.
\end{abstract}

Keywords: Demographics, Financial Behaviour, Financial Literacy, Salaried Women

JEL classification: D14, G40, G41, G50, G53

\section{Introduction}

With an immense increase in uncertainties in the financial system globally combined with the increased availability of goods and services, major risks have also risen. For every nation be developing or developed, it has therefore become vital to make its population aware of the financial products/services available, but also of the constructs on which they are based and the practical difficulties, thus making them financially literate. For women, financial literacy has become all the more crucial because they have been deemed quite fragile when it comes to financial matters $\stackrel{1}{\text {. }}$

In an age where financial products are diverse and sophisticated too, it's crucial to make informed decisions that are more relevant to financial priorities and needs for individuals to improve a comprehensive understanding of financial environments $\mathrm{s}^{2}$. The financial awareness among all levels of society facilitates the enhancement of a nation's economy. Financial literacy is one of the principal issues in stimulating the economy. Achieving financial solvency for people will help to widen the economy by allowing residents to make financially responsible decisions ${ }^{3}$. Financial literacy is the ability of individuals or organizations to manage personal financial capital through their experience and implementation ${ }^{4}$.

Over time, there has been a tremendous growth in both female education and working strength. More and more women are focussing on entrepreneurship ${ }^{5}$. Today the number of women CEOs in Fortune 500 companies is highest. Women receive more bachelor degrees than men do. More working mothers are the primary or sole earners in their family. The list is going on and on. These extraordinary facets are what make the implications of this study so incredible. The 2019 Women, Wealth, and Power Study commissioned by Allianz Life Insurance ${ }^{6}$ found that while women's empowerment has always been the subject of the past couple of years, women are continuing to make progress with financial literacy. Similarly, as previous research has shown, women are nearly three times more likely than men to admit that they may not be able to afford to save for retirement, and 
their financial literacy rates are significantly lower $\underline{7, \underline{8}}$. In addition, women relatively get paid less, invest less, and live longer, but still are liable for about the same living expenses that males pay and as they live longer, they incur extra costs, including more premiums for long-term and total health care ${ }^{9,10}$. Additionally, the primary explanation for women's reliance on men at any family life stage, whether prior to or after marriage, is financial illiteracy or lack of knowledge of finance. When it comes to global financial literacy, $70 \%$ of females were illiterate, contrary to $65 \%$ of males. In the light of the results of the Standard \& Poor's ${ }^{11}$ Ratings Services Global Financial Literacy Survey 2015, this imbalance is worse in India, with 80 percent of women compared to 73 percent of men being financially illiterate. The work of Allgood and Walstad 12 depicted that, both real and perceived financial literacy have significant impact on financial behaviour. Financial patterns, in general, include long-term planning ${ }^{13,14}$, individual retirement decisions $s^{15}$ or retirement planning ${ }^{16}$ and saving behaviour/activity $\underline{17,18}$.

Based on the conceptual research model as discussed in Figure 1, the objectives of the study based on the universe and sample studied during the period of October 2020 - January 2021 have been outlined as: i. To assess the financial literacy levels of salaried women in Ludhiana.

ii. To examine the effect of financial literacy on financial behaviour in Ludhiana among salaried women.

iii. To study the association of the demographics with level of financial literacy and financial behaviour among the salaried women in Ludhiana.

The conceptual research model of the study has been as follows:

\section{Literature Review}

Financial literacy as characterised by three variables (financial awareness, financial action, and attitude) was evaluated by the research conducted by the International Financial Education Network of the OECD in 14 countries covering four continents Atkinson and Messy ${ }^{8}$. The findings showed that there was a significant percentage of the population who lacked financial literacy in each of the surveyed countries. A low comprehension level has been identified when it comes to issues related to diversification and compound interest. In most countries, women have

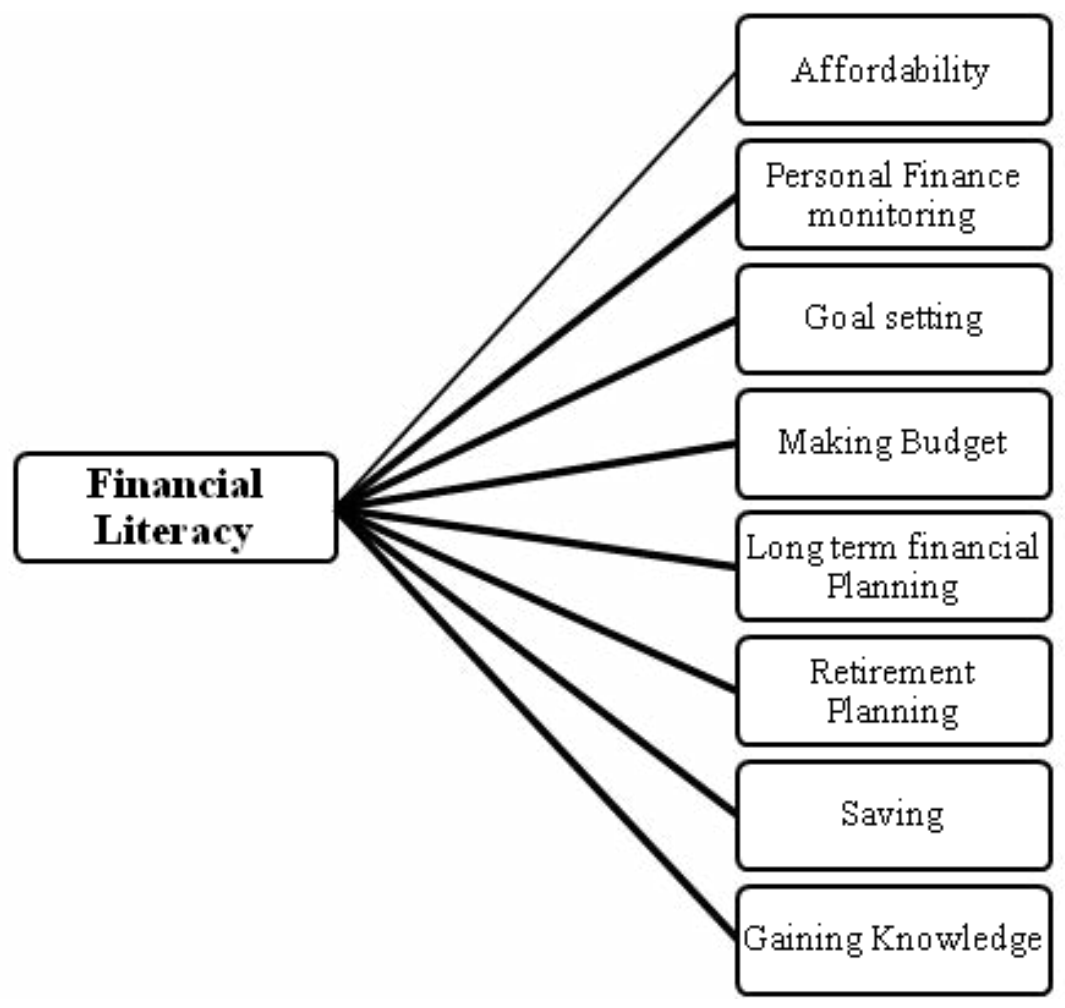

Financial Behaviour

Figure 1. Research Model. 
a lower degree of financial literacy than men. It was concluded that there was a strong positive association between financial conduct and financial literacy.

The study conducted by the NCFE 2015 on financial literacy and financial inclusion in India has been reviewed by Dwivedi et al. ${ }^{19}$ on the grounds of the profession, geographic region, and gender mix. The research by NCFE has determined that perhaps the urban population is far more financially literate than that of the rural population. Men were also found to be financially more literate than women. In contrast, the study by Dwivedi et al. ${ }^{19}$ found that women have a higher financial attitude but less financial behaviour as well as less financial knowledge, whereas men have a slightly lower financial attitude than women, but have performed better on financial behaviour and financial knowledge. Shobha and Shalini ${ }^{20}$ conducted a survey on women's understanding of personal financial planning. The study concluded that Indian women give more attention to family and child needs rather than their financial needs and individual financial safety requirements. Research has found that women, in general, assume that long-term investment choices are gold, real estate, bank deposits, insurance products, and, conversely, that women lack knowledge of modern investment instruments, such as exchangetraded funds (ETFs), options, and certificates of deposit (CDs).

Extensive studies have explored the correlation between financial literacy and financial behaviour. There are also researchers $\mathrm{s}^{21-24}$ who produced the literature on financial literacy to look into the relation between financial literacy and retirement planning. In terms of investment choices, financially literate individuals often opted for lower borrowing interest rates and have been more aware of their sufficient debt tolerance ability ${ }^{7,25}$. They end up getting cheaper mortgage loan options ${ }^{26}$ as well asless credit card loan debt crisis ${ }^{27}$. In addition to having improved retirement money, financially knowledgeable people also tend to be more able to invest in stocks, thereby creating more diverse portfolios $s^{24,28-30}$. Additionally, studies have linked financial knowledge and investment behaviour ${ }^{31}$, risk management ${ }^{\frac{32}{2}}$ and responsible financial behaviour ${ }^{33}$. However, some studies (Hilgert and Hogarth, 2003; Perry and Morris, 2005; Howlett et al., 2008; Robb and Sharpe, 2009; Mandell and Klein, 2009) $)^{34-38}$ on association among financial behaviour and financial literacy have achieved outcomes contradictory to aforesaid. Moreover, studies, such as Delafrooz and Paim ${ }^{\frac{39}{}}$, have suggested substantial variations in financial behaviour in terms of age, schooling, income and financial literacy.

\section{Research Methodology}

For assessing the level of financial literacy among salaried women, primary data was collected in form of a structured questionnaire on varied parameters (discussed in measures/scales section). Information on various demographics was gathered, besides to that on the key questions relating to financial aspects and financial behaviour. Secondary data sources used include journals such as Asia Pacific Journal of research, Economic Challenger, Economic Enquiry, Financial Services Review, Journal of Banking \& Finance, Management Finance letters (as outlined in detail in references); magazines including Outlook India, Forbes and newspapers including The Tribune, The Hindu.

Sampling: The sample size consists of 200 salaried women from Ludhiana studied during the period October 2020 - January 2021.

\subsection{Demographic Profile}

Table 1 summarises the demographic data of the respondents, as evidenced by the observation that the sample has been classified into five age groups with the highest proportion (35.5\%) of respondents from 31-35 age group. In terms of family life stage amongst four categories, majority (34\%) belong single category followed closely by those from young married with children (32\%) category. Based on education on an average most (78\%) of the respondents hold post-graduate degree while only 4 percent hold diploma. The distribution of the sample in terms of occupational sector showed that more than half of the respondents have been from education sector while minimum (2\%) have been from legal sector. Equal number of respondents have been from both finance and non-finance related work profile. The respondents have been grouped into four income categories with more than 20\% each from above $₫ 40000, \bigotimes 10001-20000$ and $\bigotimes 30001-40000$ category.

\subsection{Measures/Scales}

The questionnaire contains of fifteen financial literacy measurement items and eight financial behaviour measurement items. The scoring for both the financial literacy and financial behaviour items has been done according to the OECD recommendations (Table 2). 
Table 1. Demographic Profile of Respondents

\begin{tabular}{|c|c|c|c|c|c|c|c|}
\hline & & Frequency & Percent & & & Frequency & Percent \\
\hline \multirow[t]{5}{*}{ Age Groups } & $21-25$ yrs & 20 & 9.9 & \multirow{5}{*}{$\begin{array}{c}\text { Occupational } \\
\text { Sector }\end{array}$} & Education & 104 & 52.0 \\
\hline & $26-30$ yrs & 40 & 19.7 & & Banking & 44 & 22.0 \\
\hline & $31-35$ yrs & 72 & 35.5 & & Corporate & 32 & 16.0 \\
\hline & $36-40$ yrs & 40 & 19.7 & & Legal & 4 & 2.0 \\
\hline & $41-45$ yrs & 28 & 13.8 & & Others & 16 & 8.0 \\
\hline \multirow[t]{4}{*}{$\begin{array}{l}\text { Family Life } \\
\text { Stage }\end{array}$} & Unmarried & 68 & 34.0 & \multirow[t]{2}{*}{ Work Profile } & $\begin{array}{l}\text { Finance } \\
\text { Related }\end{array}$ & 100 & 50.0 \\
\hline & $\begin{array}{c}\text { Young } \\
\text { married } \\
\text { couple with } \\
\text { no children }\end{array}$ & 16 & 8.0 & & $\begin{array}{c}\text { Non-Finance } \\
\text { Related }\end{array}$ & 100 & 50.0 \\
\hline & $\begin{array}{c}\text { Young } \\
\text { married } \\
\text { couple with } \\
\text { children }\end{array}$ & 64 & 32.0 & \multirow[t]{4}{*}{$\begin{array}{c}\text { Work } \\
\text { Experience }\end{array}$} & Less than $5 y$ rs & 64 & 32.0 \\
\hline & $\begin{array}{l}\text { Middle-aged } \\
\text { married } \\
\text { couple with } \\
\text { children }\end{array}$ & 52 & 26.0 & & $6-10$ yrs & 72 & 36.0 \\
\hline \multirow{4}{*}{$\begin{array}{l}\text { Academic } \\
\text { Qualification }\end{array}$} & Diploma & 8 & 4.0 & & $11-15$ yrs & 44 & 22.0 \\
\hline & $\begin{array}{c}\text { Under } \\
\text { Graduation }\end{array}$ & 24 & 12.0 & & $\begin{array}{c}\text { More than } 15 \\
\text { yrs }\end{array}$ & 20 & 10.0 \\
\hline & $\begin{array}{c}\text { Post- } \\
\text { Graduation }\end{array}$ & 156 & 78.0 & \multirow[t]{5}{*}{$\begin{array}{l}\text { Monthly } \\
\text { Salary }\end{array}$} & Up to $\otimes 10000$ & 20 & 10.0 \\
\hline & Doctorates & 12 & 6.0 & & \10001-20000 & 48 & 24.0 \\
\hline & & & & & 邓20001-30000 & 36 & 18.0 \\
\hline & & & & & 邓30001-40000 & 44 & 22.0 \\
\hline & & & & & Above $\otimes 40000$ & 52 & 26.0 \\
\hline & & & & \multirow[t]{2}{*}{ Tax Payment } & Yes & 156 & 78.0 \\
\hline & & & & & No & 44 & 22.0 \\
\hline
\end{tabular}


Table 2. Scoring Methodology (OECD recommendation)

\begin{tabular}{|l|l|}
\hline $\begin{array}{l}\text { Questions asked in the questionnaire } \\
\text { to examine the Financial Literacy of the } \\
\text { Respondent }\end{array}$ & Scoring \\
\hline Correct Answer & One Mark \\
\hline Incorrect/Don't Know Answer & Zero Mark \\
\hline $\begin{array}{l}\text { Questions asked in the questionnaire to } \\
\text { examine the Financial Behaviour of the } \\
\text { Respondent }\end{array}$ & Scoring \\
\hline Strongly Agree/Agree & One Mark \\
\hline Otherwise & Zero Mark \\
\hline
\end{tabular}

\subsection{Reliability Analysis}

In order to ensure that the instrument is reliable, the reliability test has been conducted on the basis of the coefficient calculated using the Cronbach's Alpha ${ }^{1}$. For Cronbach alpha, the acceptable value is 0.7 (Hair et al., 2018) ${ }^{40}$. Table 3 indicated that the Cronbach Alpha coefficient was greater than 0.7 , reflecting the reliability of measurement tests.

Table 3. Reliability Statistics

\begin{tabular}{|l|c|c|}
\hline & Cronbach's Alpha & No. of Items \\
\hline Financial Literacy & .794 & 15 \\
\hline Financial Behaviour & .753 & 8 \\
\hline
\end{tabular}

\subsection{Financial Literacy (Descriptive Statistics)}

The descriptive analysis for the financial literacy score has been displayed in Table 4. The average total score of respondents has been 10.20 (out of 15), i.e., 68 per cent. The median score has been used to further classify respondents into subgroups, i.e., those above median, i.e., 73.33 per cent were classified as high financial literacy respondents, those equivalent to median scores as moderate financial literacy respondents, and finally those below median scores as low financial literacy respondents.
As Figure 2 illustrates, 42 percent of respondents had a high financial literacy level, 48 percent had a lower financial literacy level, and 10 percent had a mild (moderate) financial literacy level. It is clear that a higher percentage of respondents have less financial literacy. Ironically, the mean overall score of the study was higher than the OECD report on adult financial literacy. (OECD, $2020)^{41}$.

Table 4. Financial Literacy Score (Descriptives)

\begin{tabular}{|l|l|c|}
\hline & Valid & 200 \\
\cline { 2 - 3 } & Missing & 0 \\
\hline Mean & 10.20 \\
\hline Median & 11.00 \\
\hline Std. Deviation & 3.697 \\
\hline
\end{tabular}

\subsection{Financial Behaviour}

The study listed financial behaviour dimensions and assessed them on the basis of the agreeability attributed to them by the respondents using the Likert 5-point rating scale. These include affordability, personal finance monitoring, goal setting, making budget, financial planning, retirement preparedness, saving habit and knowledge seeking.

${ }^{1}$ Cronbach Alpha is a measure of internal consistency. It is considered to be a measure of scale reliability. It can be written as a function of the number of test items and the average inter-correlation among the items. 


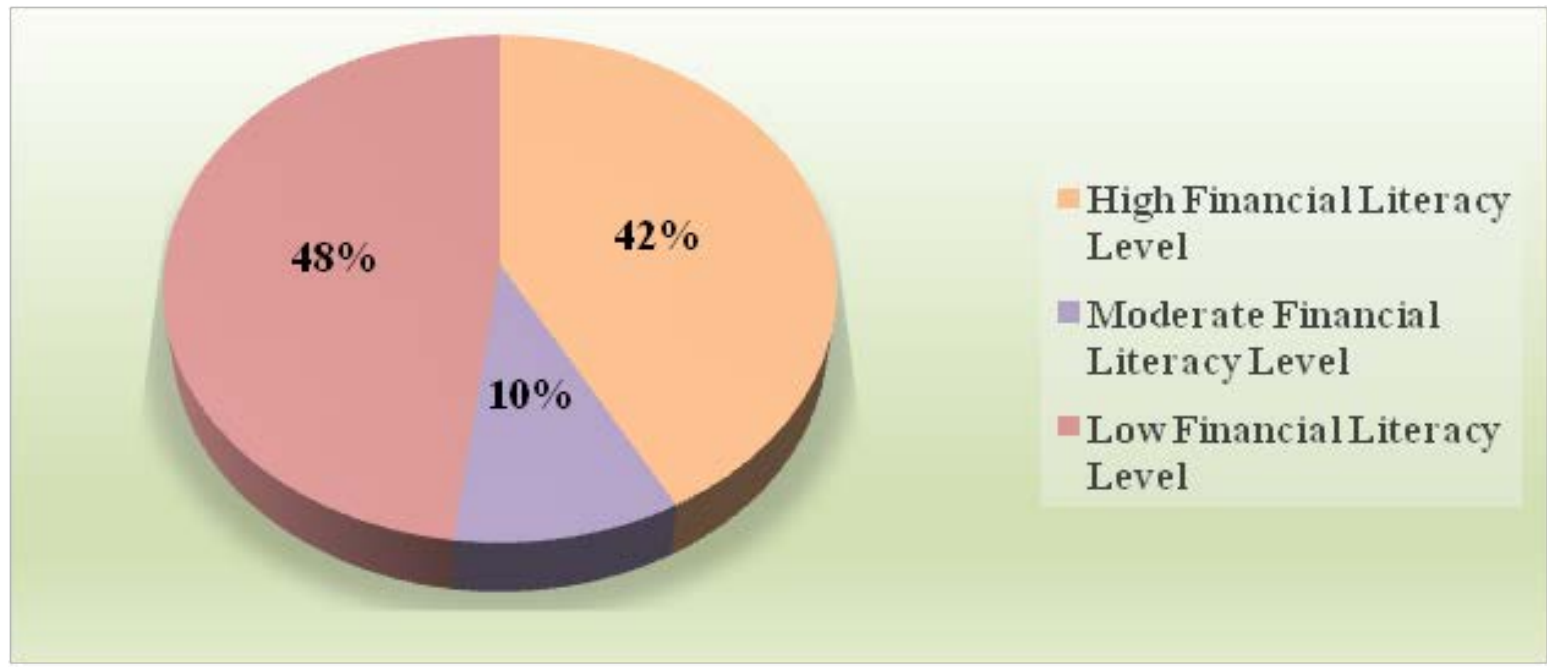

Figure 2. Financial Literacy Levels.

\subsubsection{Financial Behaviour Descriptive Statistics}

As shown in Table 5, the descriptive study show that the overall total financial behaviour score is 6.20. (out of 8 ). Based on the financial behaviour displayed by respondents, the median score has been used to stratify the respondents. As shown in Figure 3, it is apparent that 30 per cent of respondents had positive financial behaviour, while 46 per cent had negative financial behaviour, and 24 per cent had neutral financial behaviour.

Table 5. Financial Behaviour Score (Descriptives)

\begin{tabular}{|l|l|l|}
\hline \multirow{N}{*}{ Mean } & Valid & 200 \\
\cline { 2 - 3 } & Missing & 0 \\
\hline Median & 6.20 \\
\hline Std. Deviation & 7.00 \\
\hline
\end{tabular}

\subsection{Financial Literacy and Financial Behaviour}

$\mathbf{H}_{0}$ : There is no significant impact on the financial behaviour of that of financial literacy level.

$\mathbf{H}_{1}$ : There is a significant impact on the financial behaviour of that of financial literacy level.

The effect of financial literacy on the respondents' financial behaviour has been analysed by linear regression using financial literacy as an independent variable and dependent variables defined by factor analysis, represented by SPSS 26 .

\subsubsection{Factor Analysis}

The KMO value of 0.706 shown in Table 6 , along with the Bartlett test result of 0.000 , lend credence to the fact that factor analysis is a sound method.

Table 7 reflected the variables listed as representing the financial behaviour of the respondents. In total, according to the acceptable loading factor (greater than 0.5, Hair et al, 2018) ${ }^{40}$, two factors have been identified and named accordingly.

Table 6. KMO and Bartlett's Test

\begin{tabular}{|l|l|l|}
\hline \multicolumn{2}{|l|}{$\begin{array}{l}\text { Kaiser-Meyer-Olkin Measure of Sampling } \\
\text { Adequacy. }\end{array}$} & .706 \\
\hline $\begin{array}{l}\text { Bartlett's Test of } \\
\text { Sphericity }\end{array}$ & Approx. Chi-Square & 386.056 \\
\cline { 2 - 3 } & Df & 28 \\
\cline { 2 - 3 } & Sig. & $\mathbf{. 0 0 0}$ \\
\hline
\end{tabular}

Table 7. Rotated Component Matrix ${ }^{a}$

\begin{tabular}{|l|l|c|c|}
\hline \multirow{2}{*}{ Factors } & \multirow{2}{*}{ Components } & \multicolumn{2}{|c|}{ Component } \\
\cline { 2 - 4 } & & $\mathbf{1}$ & $\mathbf{2}$ \\
\hline \multirow{2}{*}{$\begin{array}{l}\text { Factor 1 } \\
\text { \& } \\
\text { Planning }\end{array}$} & Saving (FB7) & $\mathbf{. 7 0 8}$ & \\
\cline { 2 - 4 } & Seeking Knowledge (FB8) & $\mathbf{. 6 7 7}$ & \\
\cline { 2 - 4 } & Financial Planning (FB5) & $\mathbf{. 6 6 8}$ & \\
\cline { 2 - 4 } & Retirement Planning (FB6) & $\mathbf{. 5 6 5}$ & \\
\hline \multirow{3}{*}{$\begin{array}{l}\text { Factor 2 } \\
\text { Proactive } \\
\text { financial }\end{array}$} & Affordability (FB1) & & $\mathbf{. 7 5 7}$ \\
\cline { 2 - 4 } & Making Budget (FB4) & $\mathbf{. 6 6 1}$ \\
\cline { 2 - 4 } & Goal Setting (FB3) & $\mathbf{. 5 9 5}$ \\
\cline { 2 - 4 } & Personal Finance Monitoring (FB2) & & $\mathbf{. 5 9 1}$ \\
\hline \multirow{2}{*}{$\begin{array}{l}\text { Extraction } \\
\text { Rotation Method: "Principal Component Analysis". }\end{array}$} \\
\hline
\end{tabular}




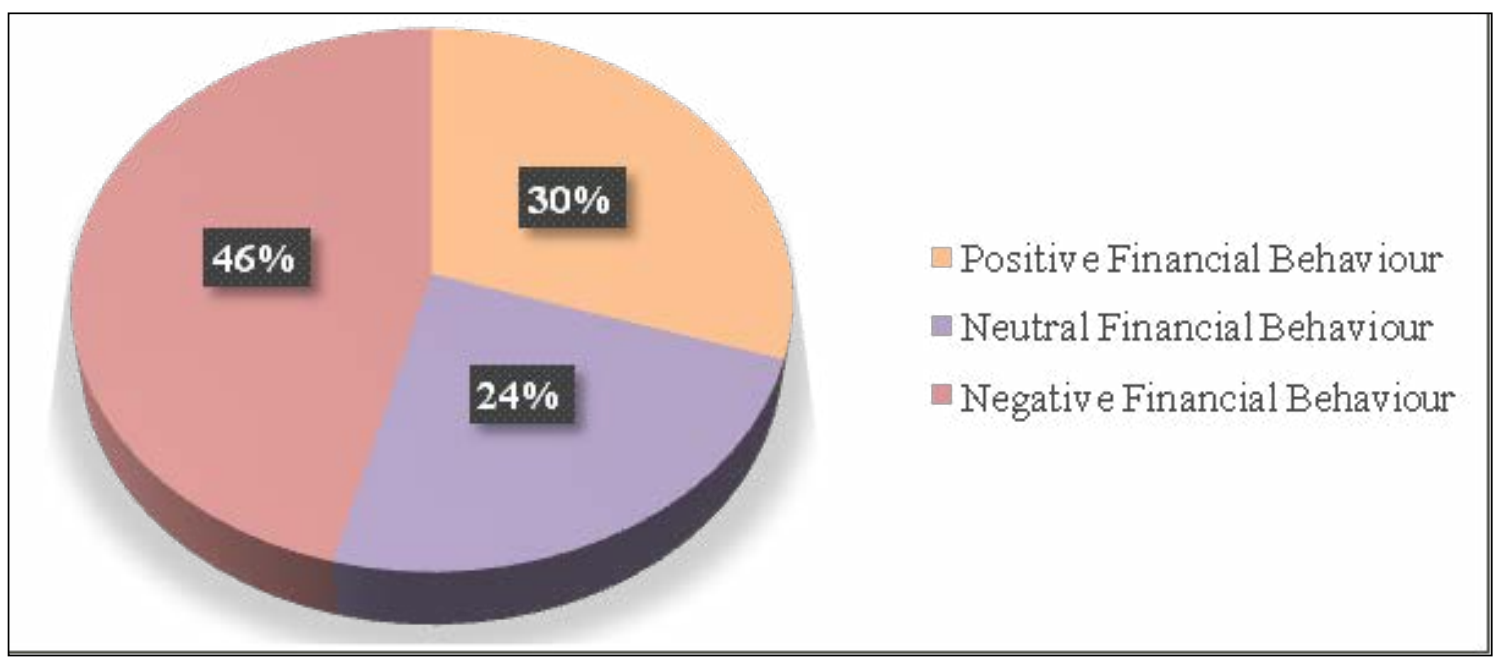

Figure 3. Financial Behaviour.

\subsubsection{Correlation and Regression Analysis}

The correlation analysis between the variables is measured using Pearson's correlation analysis. The findings have been shown in Table 8. The results revealed that the level of financial literacy among respondents has been positively and statistically significantly correlated with all financial behaviour factors (as defined through factor analysis) and the overall financial behaviour of the respondents.

The hypothesised relationship between financial literacy and financial behaviour has been specified for the current study as:

$$
\mathbf{F B}=\boldsymbol{\alpha}+\boldsymbol{\beta E}+\dot{\boldsymbol{\varepsilon}}
$$

where, FB, the dependent variable denotes Financial Behaviour; E, the independent variable denotes Financial
Literacy Level; $\alpha$ is the 'constant term' and $\beta$ is the 'coefficient of variable E i.e., Financial Literacy level.

In both cases, the t-test and F-test values (Table 9) with a p-value of less than 0.05 confirmed that the respondents' financial literacy levels had been a significant variable in explaining the variation in the respondents' financial behaviour.

The linear regression equation can be written as follows:

\section{Overall Financial Behaviour $=0.503+0.453$ \\ (Financial Literacy Level)}

The null hypothesis has been dismissed since, at a 5 percent significance level, the significant value is less than 0.05 . It is therefore estimated that the degree of financial

Table 8. Correlation Matrix

\begin{tabular}{|c|c|c|c|c|c|}
\hline & & $\begin{array}{c}\text { Financial Literacy } \\
\text { Level }\end{array}$ & $\begin{array}{c}\text { Factor } 1 \\
\text { Learning \& } \\
\text { Planning } \\
\end{array}$ & $\begin{array}{c}\text { Factor } 2 \\
\text { Proactive financial }\end{array}$ & $\begin{array}{c}\text { Overall Financial } \\
\text { Behaviour }\end{array}$ \\
\hline \multirow{3}{*}{$\begin{array}{l}\text { Financial Literacy } \\
\text { Level }\end{array}$} & Pearson Correlation & 1 & $.377^{* *}$ & $.322^{* *}$ & $.410^{* *}$ \\
\hline & Sig. (2-tailed) & & .000 & .000 & .000 \\
\hline & $\mathrm{N}$ & 200 & 200 & 200 & 200 \\
\hline \multirow{3}{*}{$\begin{array}{l}\text { Factor } 1 \\
\text { Learning \& Planning }\end{array}$} & Pearson Correlation & $.377^{* *}$ & 1 & $.462^{* *}$ & $.867^{* *}$ \\
\hline & \begin{tabular}{|l|} 
Sig. (2-tailed) \\
\end{tabular} & .000 & & .000 & .000 \\
\hline & $\mathrm{N}$ & 200 & 200 & 200 & 200 \\
\hline \multirow{3}{*}{$\begin{array}{l}\text { Factor } 2 \\
\text { Proactive financial }\end{array}$} & Pearson Correlation & $.322^{* *}$ & $.462^{* *}$ & 1 & $.842^{* *}$ \\
\hline & Sig. (2-tailed) & .000 & .000 & & .000 \\
\hline & $\mathrm{N}$ & 200 & 200 & 200 & 200 \\
\hline \multirow{3}{*}{$\begin{array}{l}\text { Overall Financial } \\
\text { Behaviour }\end{array}$} & Pearson Correlation & $.410^{* *}$ & $.867^{* *}$ & $.842^{* *}$ & 1 \\
\hline & Sig. (2-tailed) & .000 & .000 & .000 & \\
\hline & $\mathrm{N}$ & 200 & 200 & 200 & 200 \\
\hline
\end{tabular}

Note: ${ }^{*}$ Significant at the 0.01 level (2-tailed). 
literacy significantly affects respondents 'financial behaviour at confidence level of 95\%?

\subsection{Financial Literacy and Socio- Economic Demographics}

$\mathbf{H}_{0}$ : There lies no significant association between demographics and financial literacy levels.

$\mathbf{H}_{1}$ : There lies significant association between demographics and financial literacy levels.
The Pearson's Chi-Square analysis confirmed, as shown in Table 10, that amongst the socio-economic demographic variables, excluding tax payments, all other demographic variables had a significant link to respondents' financial literacy levels at a 5 percent significance level with a p-value lower than 0.05 . Therefore, in all cases, the null hypothesis has been dismissed, except in the case of tax payments.

Table 9. Regression Analysis

\begin{tabular}{|c|c|c|c|c|c|c|c|c|c|c|}
\hline \multirow{2}{*}{ Factors } & \multicolumn{2}{|c|}{$\begin{array}{c}\text { Unstandardised } \\
\text { Coefficients }\end{array}$} & \multirow{2}{*}{$\mathbf{R}^{2}$} & \multirow{2}{*}{ Adj. $\mathbf{R}^{2}$} & \multirow{2}{*}{$\begin{array}{l}\text { Std. } \\
\text { Error of } \\
\text { Estimate }\end{array}$} & \multirow{2}{*}{ Beta } & \multirow{2}{*}{ t-value } & \multirow{2}{*}{$\begin{array}{l}\text { Sig. } \\
\text { Level }\end{array}$} & \multirow{2}{*}{ F-value } & \multirow{2}{*}{$\begin{array}{l}\text { Sig. } \\
\text { Level }\end{array}$} \\
\hline & B & $\begin{array}{l}\text { Std. } \\
\text { Error }\end{array}$ & & & & & & & & \\
\hline $\begin{array}{l}\text { Factor } 1 \\
\text { Learning \& Planning } \\
\text { and }\end{array}$ & 1.785 & 0.226 & \multirow[t]{2}{*}{$\stackrel{\circ}{\stackrel{\circ}{N}}$} & \multirow[t]{2}{*}{0.138} & \multirow[t]{2}{*}{1.08} & \multirow[t]{2}{*}{0.377} & \multirow[t]{2}{*}{5.722} & \multirow[t]{2}{*}{0.000} & \multirow[t]{2}{*}{32.740} & \multirow[t]{2}{*}{0.000} \\
\hline Financial Literacy & 2.025 & 3.54 & & & & & & & & \\
\hline $\begin{array}{l}\text { Factor } 2 \\
\text { Proactive financial } \\
\text { and }\end{array}$ & 2.240 & 0.213 & \multirow[t]{2}{*}{$\stackrel{\circ}{\circ}$} & \multirow[t]{2}{*}{0.099} & \multirow[t]{2}{*}{1.02} & \multirow[t]{2}{*}{0.322} & \multirow[t]{2}{*}{4.790} & \multirow[t]{2}{*}{0.000} & \multirow[t]{2}{*}{22.946} & \multirow[t]{2}{*}{0.000} \\
\hline Financial Literacy & 1.60 & 0.334 & & & & & & & & \\
\hline $\begin{array}{l}\text { Overall Financial } \\
\text { Behaviour } \\
\text { and }\end{array}$ & 0.503 & 0.046 & \multirow[t]{2}{*}{$\stackrel{\circ}{\dot{\sigma}}$} & \multirow[t]{2}{*}{0.164} & \multirow[t]{2}{*}{0.219} & \multirow[t]{2}{*}{0.480} & \multirow[t]{2}{*}{6.324} & \multirow[t]{2}{*}{0.000} & \multirow[t]{2}{*}{39.992} & \multirow[t]{2}{*}{0.000} \\
\hline Financial Literacy & 0.453 & 0.072 & & & & & & & & \\
\hline
\end{tabular}

Table 10. Financial Literacy and Demographics

\begin{tabular}{|c|c|c|c|c|c|c|c|c|c|}
\hline \multirow{8}{*}{ 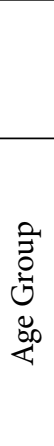 } & \multirow[b]{3}{*}{ 21-25 years } & \multicolumn{6}{|c|}{ Financial Literacy Level } & \multirow{2}{*}{$\begin{array}{c}\text { Pearson's } \\
\text { Chi- } \\
\text { Square }\end{array}$} & \multirow{2}{*}{$\begin{array}{l}\text { Sig. } \\
\text { Level }\end{array}$} \\
\hline & & \multicolumn{2}{|c|}{ High } & \multicolumn{2}{|c|}{ Moderate } & \multicolumn{2}{|c|}{ Low } & & \\
\hline & & 12 & $14 \%$ & 0 & $0 \%$ & 8 & $8 \%$ & \multirow{6}{*}{18.554} & \multirow{6}{*}{0.017} \\
\hline & 26-30 years & 16 & $19 \%$ & 4 & $20 \%$ & 20 & $21 \%$ & & \\
\hline & $31-35$ years & 32 & $38 \%$ & 8 & $40 \%$ & 32 & $33 \%$ & & \\
\hline & $36-40$ years & 8 & $10 \%$ & 8 & $40 \%$ & 24 & $25 \%$ & & \\
\hline & $41-45$ years & 16 & $19 \%$ & 0 & $0 \%$ & 12 & $13 \%$ & & \\
\hline & Total & 84 & $100 \%$ & 20 & $100 \%$ & 96 & $100 \%$ & & \\
\hline \multirow{3}{*}{ 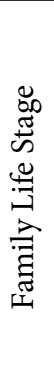 } & Unmarried & 40 & $48 \%$ & 4 & $20 \%$ & 24 & $25 \%$ & 31.131 & 0.000 \\
\hline & $\begin{array}{c}\text { Young } \\
\text { married } \\
\text { couple with } \\
\text { no children }\end{array}$ & 0 & $0 \%$ & 0 & $0 \%$ & 16 & $17 \%$ & & \\
\hline & $\begin{array}{c}\text { Young } \\
\text { married } \\
\text { couple with } \\
\text { children }\end{array}$ & 20 & $24 \%$ & 8 & $40 \%$ & 36 & $38 \%$ & & \\
\hline
\end{tabular}


Table 10. Continued

\begin{tabular}{|c|c|c|c|c|c|c|c|c|c|}
\hline & & \multicolumn{6}{|c|}{ Financial Literacy Level } & \multirow{4}{*}{$\begin{array}{c}\text { Pearson's } \\
\text { Chi- } \\
\text { Square } \\
\end{array}$} & \multirow{4}{*}{$\begin{array}{l}\text { Sig. } \\
\text { Level }\end{array}$} \\
\hline & & \multicolumn{2}{|c|}{ High } & \multicolumn{2}{|c|}{ Moderate } & \multicolumn{2}{|c|}{ Low } & & \\
\hline & $\begin{array}{l}\text { Middle-aged } \\
\text { married } \\
\text { couple with } \\
\text { children }\end{array}$ & 24 & $29 \%$ & 8 & $40 \%$ & 20 & $21 \%$ & & \\
\hline & Total & 84 & $100 \%$ & 20 & $100 \%$ & 96 & $100 \%$ & & \\
\hline \multirow{5}{*}{ 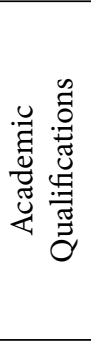 } & Diploma & 0 & $0 \%$ & 0 & $0 \%$ & 8 & $8 \%$ & \multirow{5}{*}{22.421} & \multirow{5}{*}{0.001} \\
\hline & $\begin{array}{c}\text { Under } \\
\text { Graduation }\end{array}$ & 16 & $19 \%$ & 4 & $20 \%$ & 4 & $4 \%$ & & \\
\hline & $\begin{array}{c}\text { Post- } \\
\text { Graduation }\end{array}$ & 60 & $71 \%$ & 16 & $80 \%$ & 80 & $83 \%$ & & \\
\hline & Doctorates & 8 & $10 \%$ & 0 & $0 \%$ & 4 & $4 \%$ & & \\
\hline & Total & 84 & $100 \%$ & 20 & $100 \%$ & 96 & $100 \%$ & & \\
\hline \multirow{6}{*}{ 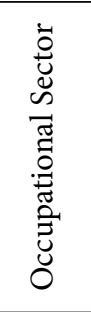 } & Education & 28 & $33 \%$ & 12 & $60 \%$ & 64 & $67 \%$ & \multirow{6}{*}{39.174} & \multirow{6}{*}{0.000} \\
\hline & Banking & 28 & $33 \%$ & 8 & $40 \%$ & 8 & $8 \%$ & & \\
\hline & Corporate & 20 & $24 \%$ & 0 & $0 \%$ & 12 & $13 \%$ & & \\
\hline & Legal & 0 & $0 \%$ & 0 & $0 \%$ & 4 & $4 \%$ & & \\
\hline & Others & 8 & $10 \%$ & 0 & $0 \%$ & 8 & $8 \%$ & & \\
\hline & Total & 84 & $100 \%$ & 20 & $100 \%$ & 96 & $100 \%$ & & \\
\hline \multirow{3}{*}{ 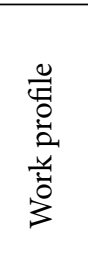 } & $\begin{array}{c}\text { Finance- } \\
\text { Related }\end{array}$ & 56 & $67 \%$ & 16 & $80 \%$ & 28 & $29 \%$ & \multirow{3}{*}{33.200} & \multirow{3}{*}{0.000} \\
\hline & $\begin{array}{c}\text { Non-Finance } \\
\text { Related }\end{array}$ & 28 & $33 \%$ & 4 & $20 \%$ & 68 & $71 \%$ & & \\
\hline & Total & 84 & $100 \%$ & 20 & $100 \%$ & 96 & $100 \%$ & & \\
\hline \multirow{5}{*}{ 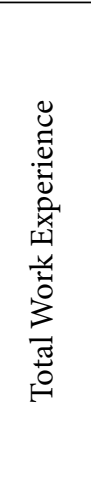 } & $\begin{array}{c}\text { Less than } 5 \\
\text { years }\end{array}$ & 32 & $38 \%$ & 0 & $0 \%$ & 32 & $33 \%$ & \multirow{5}{*}{27.955} & \multirow{5}{*}{0.000} \\
\hline & $6-10$ years & 28 & $33 \%$ & 16 & $80 \%$ & 28 & $29 \%$ & & \\
\hline & 11-15 years & 12 & $14 \%$ & 4 & $20 \%$ & 28 & $29 \%$ & & \\
\hline & $\begin{array}{c}\text { More than } \\
15 \text { yeas }\end{array}$ & 12 & $14 \%$ & 0 & $0 \%$ & 8 & $8 \%$ & & \\
\hline & Total & 84 & $100 \%$ & 20 & $100 \%$ & 96 & $100 \%$ & & \\
\hline \multirow{4}{*}{ 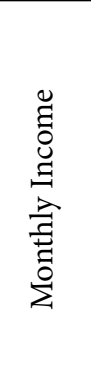 } & $\begin{array}{c}\text { Up to } \\
\text { } 10000\end{array}$ & 4 & $5 \%$ & 4 & $20 \%$ & 12 & $13 \%$ & \multirow{4}{*}{38.070} & \multirow{4}{*}{0.000} \\
\hline & $\begin{array}{c}\otimes 10001- \\
20000\end{array}$ & 12 & $14 \%$ & 4 & $20 \%$ & 32 & $33 \%$ & & \\
\hline & $\begin{array}{c}\bigotimes 20001- \\
30000\end{array}$ & 24 & $29 \%$ & 8 & $40 \%$ & 4 & $4 \%$ & & \\
\hline & $\begin{array}{c}\text { 邓30001- } \\
40000\end{array}$ & 20 & $24 \%$ & 0 & $0 \%$ & 24 & $25 \%$ & & \\
\hline
\end{tabular}


Table 10. Continued

\begin{tabular}{|c|c|c|c|c|c|c|c|c|c|}
\hline & & \multicolumn{6}{|c|}{ Financial Literacy Level } & \multirow{2}{*}{$\begin{array}{c}\text { Pearson's } \\
\text { Chi- } \\
\text { Square }\end{array}$} & \multirow{2}{*}{$\begin{array}{l}\text { Sig. } \\
\text { Level }\end{array}$} \\
\hline & & \multicolumn{2}{|c|}{ High } & \multicolumn{2}{|c|}{ Moderate } & \multicolumn{2}{|c|}{ Low } & & \\
\hline & $\begin{array}{c}\text { Above } \\
\text { \40000 }\end{array}$ & 24 & $29 \%$ & 4 & $20 \%$ & 24 & $25 \%$ & & \\
\hline & Total & 84 & $100 \%$ & 10 & $50 \%$ & 96 & $100 \%$ & & \\
\hline \multirow{3}{*}{ 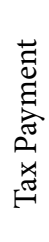 } & Yes & 68 & $81 \%$ & 12 & $60 \%$ & 76 & $79 \%$ & \multirow{3}{*}{4.279} & \multirow{3}{*}{0.118} \\
\hline & No & 16 & $19 \%$ & 8 & $40 \%$ & 20 & $21 \%$ & & \\
\hline & Total & 84 & $100 \%$ & 10 & $50 \%$ & 96 & $100 \%$ & & \\
\hline
\end{tabular}

\subsection{Financial Behaviour and Socio- Economic Demographics}

$\mathbf{H}_{0}$ : There lies no significant relationship among demographic variables and financial behaviour.

$\mathbf{H}_{1}$ : There lies significant relationship among demographic variables and financial behaviour.

The analysis for the association between financial behaviour factors (as representing financial behaviour) as identified in Table 7 and demographic variables has been done through One-Way ANOVA. As displayed in Table
11 , there lies a statistically significant association between both the financial behaviour factors (as representing financial behaviour) and Age, Family Life Stage, Total Work Experience and Monthly Income, while Academic Qualifications and Work Profile of the respondents have been statistically significantly associated with only 'Proactive Financial' behaviour factor. However, there is no significant association between both the financial factors (as representing financial behaviour) and Occupational Sector and Tax Payment of/by the respondents respectively.

Table 11. Financial Behaviour Mean Scores

\begin{tabular}{|c|c|c|c|}
\hline \multirow{2}{*}{\multicolumn{2}{|c|}{ Demographics }} & \multicolumn{2}{|c|}{ Financial Behaviour Factors } \\
\hline & & \multirow{2}{*}{$\begin{array}{c}\text { Learning \& Planning } \\
3.0000\end{array}$} & \multirow{2}{*}{$\begin{array}{c}\text { Proactive financial } \\
3.2000 \\
\end{array}$} \\
\hline & Total Sample & & \\
\hline \multirow{7}{*}{ 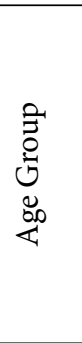 } & $21-25$ years & 3.4000 & 2.6000 \\
\hline & $26-30$ years & 1.7000 & 2.7000 \\
\hline & $31-35$ years & 3.3889 & 3.6111 \\
\hline & $36-40$ years & 3.5000 & 3.4000 \\
\hline & $41-45$ years & 2.8571 & 3.0000 \\
\hline & F-Value & 25.023 & 7.825 \\
\hline & Significance & 0.000 & 0.000 \\
\hline \multirow{6}{*}{ 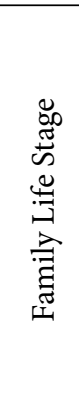 } & Unmarried & 2.6471 & 3.0000 \\
\hline & $\begin{array}{l}\text { Young married couple with no } \\
\text { children }\end{array}$ & 2.2500 & 3.0000 \\
\hline & $\begin{array}{c}\text { Young married couple with } \\
\text { children }\end{array}$ & 3.3750 & 3.6875 \\
\hline & $\begin{array}{l}\text { Middle-aged married couple } \\
\text { with children }\end{array}$ & 3.2308 & 2.9231 \\
\hline & F-Value & 7.869 & 7.037 \\
\hline & Significance & 0.000 & 0.000 \\
\hline
\end{tabular}


Table 11. Continued

\begin{tabular}{|c|c|c|c|}
\hline \multirow{2}{*}{\multicolumn{2}{|c|}{ Demographics }} & \multicolumn{2}{|c|}{ Financial Behaviour Factors } \\
\hline & & \multirow{2}{*}{$\begin{array}{c}\text { Learning \& Planning } \\
3.0000\end{array}$} & \multirow{2}{*}{$\begin{array}{c}\text { Learning \& Planning } \\
3.2000\end{array}$} \\
\hline & Total Sample & & \\
\hline \multirow{6}{*}{ 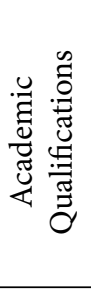 } & Diploma & 2.0000 & 2.0000 \\
\hline & Under Graduation & 3.0000 & 3.6667 \\
\hline & Post-Graduation & 3.0513 & 3.2564 \\
\hline & Doctorates & 3.0000 & 2.3333 \\
\hline & F-Value & 2.085 & 8.338 \\
\hline & Significance & 0.104 & 0.000 \\
\hline \multirow{7}{*}{ 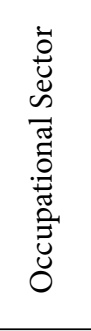 } & Education & 3.0769 & 3.2308 \\
\hline & Banking & 2.9091 & 3.4545 \\
\hline & Corporate & 3.0000 & 3.0000 \\
\hline & Legal & 4.0000 & 3.0000 \\
\hline & Others & 2.5000 & 2.7500 \\
\hline & F-Value & 1.664 & 1.658 \\
\hline & Significance & 0.160 & 0.161 \\
\hline \multirow{4}{*}{ 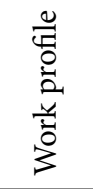 } & Finance-Related & 3.1600 & 3.3600 \\
\hline & Non-Finance Related & 2.8400 & 3.0400 \\
\hline & F-Value & 3.799 & 4.468 \\
\hline & Significance & 0.053 & 0.036 \\
\hline \multirow{6}{*}{ 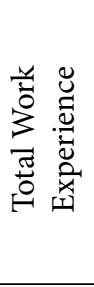 } & Less than 5 years & 2.6250 & 2.8125 \\
\hline & $6-10$ years & 3.1667 & 3.2222 \\
\hline & $11-15$ years & 3.2727 & 3.8182 \\
\hline & More than 15 yeas & 3.0000 & 3.0000 \\
\hline & F-Value & 3.618 & 8.699 \\
\hline & Significance & 0.014 & 0.000 \\
\hline \multirow{7}{*}{ 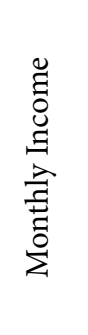 } & Up to $\bigotimes 10000$ & 3.0000 & 2.8000 \\
\hline & \10001-20000 & 2.3333 & 2.8333 \\
\hline & $\triangle 20001-30000$ & 3.1111 & 3.5556 \\
\hline & $\bigotimes 30001-40000$ & 3.5455 & 3.4545 \\
\hline & Above $\bigotimes 40000$ & 3.0769 & 3.2308 \\
\hline & F-Value & 7.241 & 3.880 \\
\hline & Significance & 0.000 & 0.005 \\
\hline \multirow{4}{*}{ 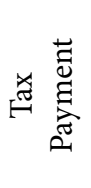 } & Yes & 2.9744 & 3.2308 \\
\hline & No & 3.0909 & 3.0909 \\
\hline & F-Value & 0.340 & 0.575 \\
\hline & Significance & 0.561 & 0.449 \\
\hline
\end{tabular}

\section{Conclusion}

Globally, the current scenario is characterised by prodigious shifts in the financial sector with the emergence of a huge range of investment opportunities, but these paths, on the one hand, offer higher returns and, on the other, contribute to higher levels of risk and uncertainty.
It has therefore become imperative for individuals of all age groups, particularly those belonging to financially vulnerable groups $s^{42}$, such as women, to be aware of the principles on which these avenues are centered. This will enable them to make best choices, especially the earning women so that they can utilise their earnings in a way that can ensure their current and future financial security 
thus making them economically empowered. Although the average financial literacy score of the entire sample is higher than that of $\mathrm{OECD}^{41}$ score, still majority of the respondents possessed low financial literacy levels, thereby highlighting the need for the policy makers, government to take adequate initiatives in this regard.

In addition, the majority of respondents demonstrated negative financial behaviour, thus weakening the capacity of women to control their finances. As regards to the relationship between the financial literacy levels and demographics, significant associations were found between the respondents' age, family life stage, academic qualifications, occupational sector, work profile, overall work experience, and monthly income, whereas no significant relationship was found between the respondents' levels of financial literacy and tax payment.

\section{Theoretical Implications}

The aim of the research was to create a substantial addition to the literature on financial literacy for salaried women. A systematic methodology has been used for the evaluation of financial literacy, which, in turn, has been shown to have a substantial effect on subsequent financial decisions. The purpose of the analysis is to aid policymakers, governments, agencies, banks, etc. by supplying them with insights further into salaried women's financial literacy and its bearing on their financial behaviour. This understanding will encourage them to develop better policies/products/services that are specifically tailored to women. These concrete, concentrated initiatives would include substantive economic empowerment and equality for women.

\section{Limitations of the Study}

The analysis for this study was confined to salaried women in Ludhiana city. The approach used for primary data collection has been the non-probability convenience sampling method, which has its own restriction, and is therefore applied automatically to the analysis. The research can be extended to include contrasts between salaried and non-salaried individuals or urban and rural areas. Research may also discuss the role of financial literacy in women's empowerment across a wide spectrum. The research is only performed for the city of Ludhiana, it can also be conducted in different parts of the region or country.

\section{References}

1. Outlook India. The Importance of Financial Literacy among Women in Today's Time. 2019. Available at https:// www.outlookindia.com/outlookmoney/talking-money/ the-importance-of-financial-literacy-among-women-intodays-time-2895

2. Kumar S \& Anees M. Financial literacy and education: Present scenario in India. International Journal of Engineering and Management Research. Dec 2013; $3(6): 83-7$.

3. Agarwalla SK, Barua SK, Jacob J, Varma JR. Financial literacy among working young in urban India. Indian Institute of Management Ahmedabad, WP. 10 Oct 2013; 10(2013-10):02-27.

4. Warsono W. Prinsip-Prinsip dan Praktik keuangan Pribadi (The Principles and Practice of Personal Finance). Jurnal Salam. Jan 11 2011; 13(2).

5. Ortiz-Ospina E, Tzvetkova S, Roser M. Women's employment. Our World in Data. Mar 242018.

6. Allianz Life, 2019. For women, rising social power is not coinciding with a rise in financial confidence. [online] Allianz Life Insurance Company of North America, p.2. Available at: <https://www.allianzlife.com/-/media/files/ allianz/pdfs/newsroom/2019-women-money-and-powersummary-sheet.pdf> [Accessed 6 December 2020].

7. Lusardi A, Tufano P. Debt literacy, financial experiences, and over indebtedness. Journal of Pension Economics \& Finance. Oct 2015; 14(4):332-68.

8. Atkinson A, Messy FA. Measuring financial literacy: Results of the OECD/International Network on Financial Education (INFE) pilot study. 2012.

9. Sabri MF, Juen TT, Othman MA, Rahim HA. Financial literacy, financial Management practices, and retirement confidence among Women working in government Agencies: A mediation model. The Journal of Developing Areas. 2015; 49(6):405-12.

10. Weiss C, Parkinson D, Duncan A. Living longer on less: women, paid work, and superannuation in Victoria, Australia. SAGE Open. Jul 24 2015; 5(3):1c12. https://doi. org/10.1177/2158244015597966.

11. Klapper L, Lusardi A, Van Oudheusden P. Financial literacy around the world. Standard \& Poor's Ratings Services Global Financial Literacy Survey. Washington: Standard \& Poor's. 2015.

12. Allgood S, Walstad WB. The effects of perceived and actual financial literacy on financial behaviors. Economic inquiry. 2016 Jan; 54(1):675-97.

13. Alhenawi Y. Financial literacy of US households: Knowledge vs. long-term financial planning. Financial services review. $2013 ; 22$. 
14. Zulaihati S, Susanti S, Widyastuti U. Teachers' financial literacy: Does it impact on financial behaviour? Management Science Letters. 2020; 10(3):653-8.

15. Clark R, Lusardi A, Mitchell OS. Employee financial literacy and retirement plan behavior: a case study. Economic Inquiry. Jan 2017; 55(1):248-59.

16. Bateman, H., Louviere, J., Thorp, S., Islam, T., \& Satchell, S. Investment decisions for retirement savings. The Journal of Consumer Affairs. 2017; 44(3):463-82.

17. Lusardi A. Financial literacy: an essential tool for informed consumer choice? National Bureau of Economic Research. Jun 192008.

18. Murendo C, Mutsonziwa K. Financial literacy and savings decisions by adult financial consumers in Zimbabwe. International journal of consumer studies. 2017 Jan; 41(1):95-103.

19. Dwivedi M, Purohit H, Mehta D. Improving financial literacy among women: The role of universities. Economic Challenger. Sep 30 October-December. 2015.

20. Shobha TS, Shalini S. A study on the perception of women towards financial planning in the city of Bengaluru. Asia Pacific Journal of Research. 2015; 1(30):14-21.

21. Ameriks J, Caplin A, Leahy J. Wealth accumulation and the propensity to plan. The Quarterly Journal of Economics. Aug 1 2003; 118(3):1007-47.

22. Lusardi A, Mitchell OS. Baby boomer retirement security: The roles of planning, financial literacy, and housing wealth. Journal of Monetary Economics. Jan 1 2007; 54(1):205-24.

23. Bucher-Koenen T, Lusardi A. Financial literacy and retirement planning in Germany. National Bureau of Economic Research. 2011 Jun 9.

24. Van Rooij M, Lusardi A, Alessie R. Financial literacy and stock market participation. Journal of Financial Economics. Aug 1 2011; 101(2):449-72.

25. Stango V, Zinman J. Exponential growth bias and household finance. The Journal of Finance. Dec 2009; 64(6):2807-49.

26. Disney R, Gathergood J. Financial literacy and consumer credit portfolios. Journal of Banking \& Finance. Jul 12013 ; 37(7):2246-54.

27. Gathergood J. Self-control, financial literacy and consumer over-indebtedness. Journal of Economic Psychology. Jun 1 2012; 33(3):590-602.

28. Christiansen C, Joensen JS, Rangvid J. Are economists more likely to hold stocks?. Review of Finance. Jan 1 2008; 12(3):465-96.
29. Guiso L, Jappelli T. The role of intuition and reasoning in driving aversion to risk, aversion to ambiguity and regret. Mimeo. 2008.

30. Abreu M, Mendes V. Financial literacy and portfolio diversification. Quantitative finance. May 1 2010; 10(5):515-28.

31. Morrin M, Broniarczyk S, Inman JJ, Broussard J. Saving for retirement: The effects of fund assortment size and investor knowledge on asset allocation strategies. Journal of Consumer Affairs. Jun 2008; 42(2):206-22.

32. Dulebohn JH, Murray B. Retirement savings behaviour of higher education employees. Research in Higher Education. Aug 2007; 48(5):545-82.

33. Grable JE, Park JY, Joo SH. Explaining financial management behaviour for Koreans living in the United States. Journal of Consumer Affairs. Mar 2009; 43(1):80107.

34. Hilgert MA, Hogarth JM, Beverly SG. Household financial management: The connection between knowledge and behaviour. Fed. Res. Bull.. 2003; 89:309.

35. Perry VG, Morris MD. Who is in control? The role of self®perception, knowledge, and income in explaining consumer financial behaviour. Journal of Consumer Affairs. Sep 2005; 39(2):299-313.

36. Howlett E, Kees J, Kemp E. The role of self冈regulation, future orientation, and financial knowledge in long冈term financial decisions. Journal of Consumer Affairs. Jun 2008; 42(2):223-42.

37. Robb CA, Sharpe DL. Effect of personal financial knowledge on college students' credit card behaviour. Journal of Financial Counselling and Planning. 2009; 20(1).

38. Mandell L, Klein LS. The impact of financial literacy education on subsequent financial behaviour. Journal of Financial Counselling and Planning. 2009; 20(1).

39. Delafrooz N, Paim LH. Determinants of financial wellness among Malaysia workers. African Journal of Business Management. 2011 Oct 14; 5(24):10092-100.

40. Joseph F. Hair, Barry J. Babin, Rolph E. Anderson, William C. Black. Multi variate data analysis. Cengage Learning India. 2018.

41. OECD. OECD/INFE 2020 international survey of adult financial literacy.

42. Financy. Women are more financially vulnerable than men. 2020. Available at https://financy.com.au/women-aremore-financially-vulnerable-than-men/ 